International Journal of Computer Science \& Information Technology (IJCSIT) Vol 3, No 4, August 2011

\title{
Packet Scheduling of two Classes Flow
}

\author{
Rabie BARHOUN ${ }^{* 1}$ and Abdelwahed NAMIR ${ }^{2}$ \\ Department of Mathematics and Computer Science, University Hassan II, \\ Mohammedia, P.O Box 7955 Sidi Otmane, Casablanca, Morocco \\ ${ }^{1}$ barhounrabiedyahoo.fr \\ 2a.namir@yahoo.fr
}

\begin{abstract}
Various techniques have been proposed to achieve good QoS for diverse application types at network nodes. In order to supply the various QoS requirements for different kinds of applications, new scheduling policies need to be developed and evaluated. Many scheduling techniques have been proposed to respond to the temporal requirements of real-time flows. We propose a new scheduling Hierarchical Hybrid EDF/FIFO for network service, which considers the real time flow have higher priority to service. The technique uses the algorithm Earliest Deadline First (EDF) because the optimality of this algorithm has been proved for a number of criteria. However, this algorithm knows a complexity that increases with the size of queue. Our approach for scheduling is nonpreemptive and based in two queues. The first queue for real time flow (class-1) combines the two policies EDF and First in First out (FIFO) to reduce the complexity of $E D F$. The second queue for non real time flow (class-2) is scheduled by FIFO policy. The work complexity of our approach is less or equal than $O\left(n \log _{2} n\right)$ with $n$ is the length of the hierarchical queue. The simulation results underline the effectiveness of this approach.
\end{abstract}

\section{KEYWORDS}

Scheduling, QoS, Flow, Non-preemtive, Real Time, Non Real Time, EDF, FIFO.

\section{INTRODUCTION}

Nowadays, many applications newly developed, such as wireless sensor networks or high speed packet switching networks rely on the ability of the network, in which the quality of service (QoS) of the applications is guaranteed. These guarantees are usually bounded in the form of delay, bandwidth, jitter and reliability [1] or a combination of these parameters. These parameters determine the QoS that is required by the. For example, a typical e-mail application has stringent requirements for reliability. Every bit has to be delivered correctly for a successful transmission. On the other hand, the e-mail application is insensitive to delay and jitter, and does not require a high Bandwidth. The user would not care less if the e-mail reaches its destination in a few milliseconds or a few seconds. However, for other types of applications these requirements can be completely different.

Furthermore, the applications that are existing in a packet network, which provide QoS guarantees on the traffic are different types, some of them are real time applications such as video traffic and VoIP with stringent QoS requirements, while other applications, non real time, no guarantees needed. Different techniques have been used by packet networks to provide QoS guarantees to the traffic use. The technique of a scheduling algorithm in a packet network is to

\footnotetext{
"Corresponding Author.

DOI : $10.5121 /$ ijcsit.2011.3410
} 
decide the packet that should be transmitted in the next cycle from the available arrived packets [2], [3].

Network traffic can be categorized into two types: real-time traffic, such as multimedia, business-critical applications, telemedicine, and non-real-time traffic such as http data, e-mail application or file transfer. Recently, there has been a significant increase in the amount of multimedia services transmitted over networks. These multimedia applications, due to the stringent delay constraints, have to meet certain QoS guarantees. Since scheduling has a direct impact on the system capacity and delay as well as throughput, it is therefore necessary to investigate the suitable scheduling algorithms for such traffic. The distinguishing characteristic of real-time traffic is that it requires bounded delay while it can tolerate some packet losses. The delay can be bounded by associating a deadline for each packet. Once a packet misses its deadline, it will be dropped as it is no longer useful. Therefore the main goal for any scheduling scheme for real-time traffic is to deliver packets meets its deadline. Many scheduling algorithms have been proposed to meet this goal. The First In First Out (FIFO) scheduling algorithm, which is mostly used in conventional networks, is widely adopted for best-effort traffic. On the other hand, many scheduling algorithms have been proposed to proved different schemes of QoS guarantees, these algorithms include Earliest Deadline First (EDF).

In this paper, we present an approach non-preemptive for scheduling two classes of flows. The class-1 has highest priority, which represents real-time and specified by absolute deadlines (where the absolute deadline is the interval of time between the arrival of a packet and its relative deadline). However, class- 2 has lower priority and represents non real-time. The class- 1 packets are served according to EDF and FIFO, while the class- 2 packets are served according to FIFO.

The article is organized as the following. Section 2 presents some techniques for scheduling queue. Section 3 describes the Hierarchical Hybrid EDF/FIFO system. The complexity of the system is studied in section 4 . Section 5 presents details of our design, and simulation approach with the corresponding results to gauge the benefits of our approach. Finally, section 6 is reserved for final conclusion and highlighting some perspective work.

\section{SCHEDULING QUEUE}

In this section we present the packet scheduling algorithms widely used for scheduling real time flow and no real time flow. Based on this, we define new policy for QoS guarantee to different applications.

\subsection{FIFO Queuing}

This is the classical scheduling algorithm deployed in the best-effort approach in the Internet and it is also known as FIFO (First In First Out). With this algorithm, the data are sent in the same order in which they are received. The complexity of this approach is very low and it is also very efficient to implement in hardware. It is a work-conserving algorithm and because its characteristics it has been adopted by a large number of network architectures. Unfortunately, FIFO has several limitations:

- It does not provide fairness;

- The support to control congestion is limited

This kind of scheduler is not suitable for stream like multimedia traffic, because it is not able to isolate real-time flow from best effort ones. In this case, there is no way to guarantee a specific level of quality to real-time flow.

As a computer revolution, many scheduling algorithms have been proposed to meet this goal. FIFO scheduling algorithm, which is mostly used in conventional networks, is widely adopted 
for best-effort traffic. This method is easy to realize and its complexity is reduced to $\mathrm{O}(1)$. On the other hand, the weak point of this method is long delay-time from Queue because every packet is processed in a single Queue, and delay time occurs in processing each single Queue.

\subsection{EDF Queuing}

Many real-time systems rely on the earliest deadline first (EDF) scheduling algorithm. This algorithm guided by deadline, represent a special attention [4], [5], [6]. Its principle is simple, that is sorting packets in order of their absolute deadline. More precisely, each flow on a deadline is representing the constraint on the response time. Thus, when a packet arrives at a node (router or switch), the EDF scheduling algorithm assigns an absolute deadline equal to its arrival time in the node plus the relative deadline of the flow which belongs. The packet with the smallest absolute deadline will be transmitted first [7], [8]. The study of the EDF scheduling is interesting, especially since this algorithm has been proved optimal mono-processor environment where the moment's activation of the packets is not known a priori [9], [10], [11]. Its implementation in real networks has been the subject of many studies [12], [13] and recently by [14], [15], [16], [17]. However, this algorithm has significant complexity deriving from an incremental cost of classification packets, which increases with the queue's length, furthermore, its efficiency becomes more consistent in case of high load.

\section{HiERARCHICAL HYBRID EDF/FIFO SYSTEM}

The larger network such as internet offering a single best effort service in which coexist the various requirements, such as business-critical applications, telemedicine, message or file transfer. To satisfy the various requirements, a uniform treatment was inappropriate. Even with a surplus of bandwidth, the demanding flows in real time (i.e. critical applications or response time) are not privileged. They can be penalized by lower priority flows.

In this paper, we propose a new scheduling algorithm Hierarchical Hybrid EDF/FIFO which can meet the real-time with complexity reduces while continuing to provide best effort service over heterogeneous network traffic. The major problem with EDF scheduler is that lower priority flows, such as Non-Real-Time traffic, can starve as it is characterized by long waiting time [18]. Despite EDF provides stable QoS guarantees to high priority flows, such as Real-Time traffic, the deadline miss rates of the low priority flows can be unacceptably high.

We can summarize the main drawbacks of the EDF scheduler when implemented in a large network where there are different applications, as follows:

- The work complexity of the EDF scheduler is important, and which becomes more pronounced when the queue length increases drastically.

- The flow Non-Real-Time still penalized by Real-Time flow that receives a high priority.

The Hybrid EDF/FIFO scheduling proposed [19] to overcome the first drawback of the EDF. This algorithm combines both the EDF and the FIFO scheduling algorithms. Idea consists in limiting the usage of EDF to the scheduling of the first k packets; and the remaining packets are scheduled via the simple and fast FIFO algorithm. Analyzing this scheduling algorithm, it is obvious that the last drawback is still discovered when Hybrid EDF/FIFO is used to schedule heterogeneous network traffic. Our approach Hierarchical Hybrid EDF/FIFO satisfies this objective. It can meet the real-time needs of such applications, by using a priority queue Hybrid EDF/FIFO, while continuing to provide best effort service to non-real time traffic by lower priority queue FIFO.

For this, we classified the flows into two classes; the first class (class-1) is a priority and represents Real Time flows (RT-F). The second class (class-2) is a lower priority and 
corresponds to Non-Real-Time flows (NRT-F). Earliest Deadline First (EDF) is on ideal scheduler for real time flows because the optimality of this algorithm has been proved for number criteria. However, this algorithm knows a complexity that increases with the size of the queue. In order to schedule the flow of class- 1 with a complexity reduces and to not penalize the flows of class-2, our scheduling approach is non preemptive and based in two queues. The first is a queue hybrid EDF/FIFO for class-1 that combines the two polices FIFO and EDF. The second queue for class- 2 is scheduled by simple FIFO policy. The complexity of this approach is less than $O\left(n \log _{2} n\right)$. The performances of the proposed approach are evaluated by the miss deadline of the packet in the hybrid queue the class- 1 and the average time of residence of packet in the FIFO queue of class-2.

\section{COMPLEXITY SySTEM}

Consider an execution of the Hierarchical Hybrid EDF/FIFO scheduling discipline over $\mathrm{n}$ packet flows. We define the scheduler work complexity's n packets for service.

Theorem: The complexity of the Hierarchical Hybrid EDF/FIFO queue is less than or equal, in the worst-case, the complexity of the EDF, i.e. is less or equal than $O\left(n \log _{2} n\right)$.

Proof. We prove this theorem by showing that the total enqueuing and dequeuing operations are each of time complexity is less than $O\left(n \log _{2} n\right)$. We start by finding the time complexity of the enqueuing operation and then the dequeuing operation. Let $r$ is the number of the packet presents in class-1 (real-time flow), and $m$ is the number of the packet presents in class-2 (non real-time flow). The time complexity of the enqueuing operation for the class- 2 is of constant order $O(1)$ since the class- 2 is enqueued by FIFO policy. On the other hand, the time complexity of the enqueuing operation for the class-1 is of order $O\left(\log _{2} k\right)$, which $k$ is the length of the portion of the hybrid queue managed by EDF policy, and the rest of the queue (length $\mathrm{r}-\mathrm{k}$ ) is managed by FIFO policy since the $k$ first packets are enquired by EDF algorithm which sorts the incoming traffic with the shortest deadline packet at the head of the queue. Consequently, this result in a very lower enqueuing total time complexity compare to the standard EDF as $k<r$ and $r<n$. Moreover, if all of the sources' packets have the same deadline, then EDF operates as FIFO with a total enqueuing time complexity of constant order $o$ (1). For the worst-case, however, the total time complexity of the enqueuing operation is defined by an order of $O\left(n \log _{2} n\right)$. This due to the fact that the worst-case might occur when all sources' traffic is of class- $1, k=r$ and then we have $r=n$ and $m=0$. To find the total time complexity of the dequeuing operation, we calculate the time complexity of the dequeuing operation for both $r$ and $m$ traffic. Since the $m$ traffic is dequeued by FIFO algorithm. Also, the time complexity of the dequeuing operation for the traffic is of constant order $O(1)+O\left(\log _{2} k\right)$ since the $r$ traffic is again dequeued by FIFO algorithm but once the packet is served, the packet of the part of manage queue by FIFO must be enqueue in part managed by EDF. Moreover, the operation of removing the selected packet for service from the head of the Hybrid EDF/FIFO queue is still can be executed in a constant order $O\left(\log _{2} k\right)$ time.

Consequently, the total time complexity is still of constant order:

$$
o(n)+o(r-k)+O\left(k \log _{2} k\right)=O(m+r-k)+o\left(k \log _{2} k\right) .
$$

Therefore, the total worst-case complexity of Hierarchical Hybrid EDF/FIFO at a scheduling event is less than or equal

$$
o(n-k)+O\left(k \log _{2} k\right) \leq o\left((n-k) \log _{2} k\right)+o\left(k \log _{2} k\right)
$$

Moreover

$$
O\left(a \log _{2} a\right)+o\left(b \log _{2} b\right) \leq o\left((a+b) \log _{2}(a+b)\right)
$$


International Journal of Computer Science \& Information Technology (IJCSIT) Vol 3, No 4, August 2011

Then, the complexity in the worst-case of our system, is less than or equal, the complexity of the $\mathrm{EDF}$, i.e. is less or equal than $O\left((n-k+k) \log _{2}(n-k+k)=O\left(n \log _{2} n\right)\right.$.

\section{SYSTEM QUEUE MANAGEMENT}

In our Hierarchical Hybrid EDF/FIFO system consists of two queues: A high priority queue with Hybrid EDF/FIFO service for the real-time traffic such as video, voice and Multimedia, and a low priority queue with FIFO service for the non-real-time traffic such as http data are implemented as the figure 1 shows.

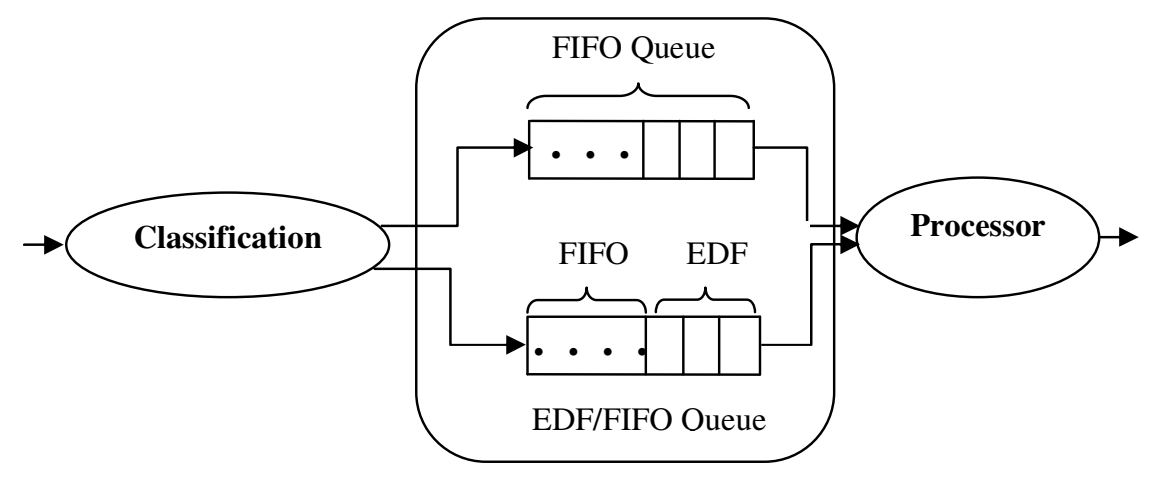

Figure 1. Structure of Hierarchical Hybrid EDF/FIFO system

\subsection{Notations and parameters}

Table 1 summarizes the internal parameters of our hierarchical scheduling algorithm.

Table. 1 Internal parameter of the Hierarchical Hybrid EDF/FIFO algorithm

\begin{tabular}{|l|l|}
\hline \multicolumn{1}{|c|}{ Parameter } & \multicolumn{1}{c|}{ Signification } \\
\hline$H$ & queue Hybrid EDF/FIFO \\
\hline$H_{z}$ & the portion of the H queue managed by EDF \\
\hline$H_{f}$ & the portion of the H queue managed by FIFO \\
\hline$F$ & the queue FIFO \\
\hline$L$ & Length of the Hierarchical Hybrid EDF/FIFO queue \\
\hline$x$ & Type of the queue (service) H or F \\
\hline$Z_{x}$ & Length of the $\mathrm{x}$ queue \\
\hline$h$ & Length of the $\mathrm{x}$ queue managed by EDF \\
\hline$n$ & Length of the $\mathrm{x}$ queue managed by FIFO \\
\hline$q_{x}$ & Number of packet in x queue \\
\hline$p$ & Packet \\
\hline$p^{x}$ & Packet of the head of \\
\hline$p_{x}$ & Packet of the tail of \\
\hline$d_{z}$ & Relative Deadline of the packet $\mathrm{p}$ \\
\hline
\end{tabular}

\subsection{Algorithm description}

In the following table (Table 2), we present a pseudo-algorithm of the Hierarchical hybrid queue implemented in a node (router or switch) for dealing with packet arrivals. When the node 
receives a packet destined to transmit, the node classifies the packet to determine whether the package belongs to the class-1or class-2. If hierarchical queue is already full, the node drops the packet. Otherwise, the node appends the packet to the corresponding queue. Besides, if the queueued packet belongs to the class- 1 , the scheduler reacts according to a hybrid algorithm that combines FIFO and EDF.

Table 2. The Hierarchical Hybrid EDF/FIFO algorithm

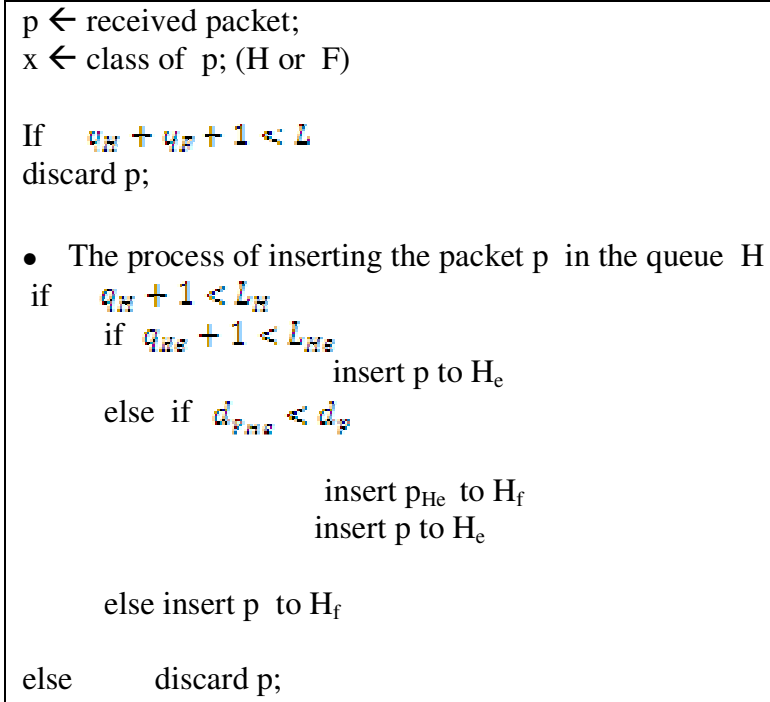

- The process of inserting the packet $\mathrm{p}$ in the queue $\mathrm{F}$

if $q_{F}+1 \leqslant L_{F}$ insert $p$ to $F$

else discard $\mathrm{p}$;

- The process of serving

$$
\text { If } c_{K}=0
$$

/* The F queue well be served */

else

$$
\begin{aligned}
& q_{s}-q_{s}-1 ; \\
& \text { Send }\left(\mathrm{p}^{\mathrm{F}}\right) ;
\end{aligned}
$$

/* The $\mathrm{H}$ queue well be served */

$$
\begin{aligned}
& q_{H}-q_{H}-1 ; \\
& \operatorname{Send}\left(\mathrm{p}^{\mathrm{H}}\right) ;
\end{aligned}
$$

We consider a network topology comprising 4 traffic sources, three of them generate real time flow (class-1) while the fourth generates non real time flow (class-2), sharing equally a $3 \mathrm{Mbps}$ link. The link is managed by three scheduling algorithms Hierarchical Hybrid EDF/FIFO, Hierarchical EDF and Hierarchical FIFO. The Buffer size is set to 100 packets for class-1 and 100 packets for class-2.

The packet size has direct impact on the system status, for that reason, the size of data packet was 1150 Byte for all class, the reason to choose this value is that almost $50 \%$ of the traffic 
being propagated across the internet has a packet size of 1150 Byte as shown in Figure 2 which was studied in [20].

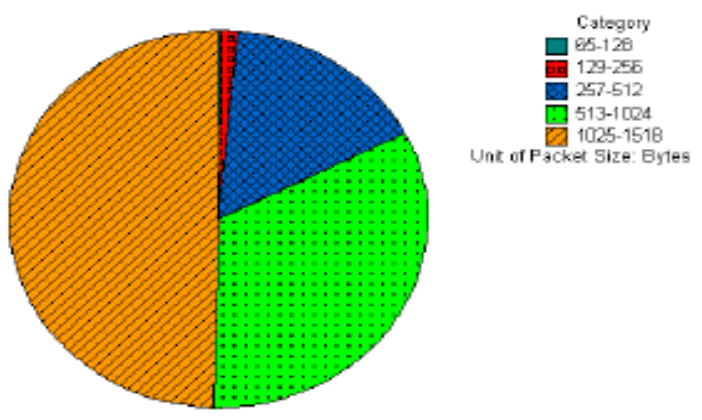

Figure 2. Packet size distribution

For the three flows of class- 1 , we consider three flowing deadlines: $(d 1=3 \mathrm{~ms}$; $d 2=50 \mathrm{~ms}$; $\mathrm{d} 3=120 \mathrm{~ms}$ ).

For all traffic, we used ON/OFF bursty traffic (i.e., a sequence of ON and OFF parts). The ON part represents a random period of time during which the source generates traffic, whereas the OFF part designates a random idle period where no traffic is sent. The exponentially distributed ON/OFF periods as used.

Three graphs were plotted to compare the performance of the three scheduling algorithms: Hierarchical FIFO, Hierarchical EDF and Hierarchical Hybrid EDF/FIFO for the different flow (class- 1 and class-2). Figures 3 and 4 show the packet percentage miss deadline of the class- 1 (for examples video, Voice and telemedicine) when using each the three scheduling algorithm. The results show that number of packets whose deadlines are met increases drastically when the size of the EDF part of the queue Hierarchical Hybrid EDF/FIFO increases. So, the number of packet miss deadline of our approach is less than the ones of the approach Hierarchical FIFO, but it approximates the ones of the Hierarchical EDF proportionately with size of the EDF's hierarchical Hybrid EDF/FIFO.

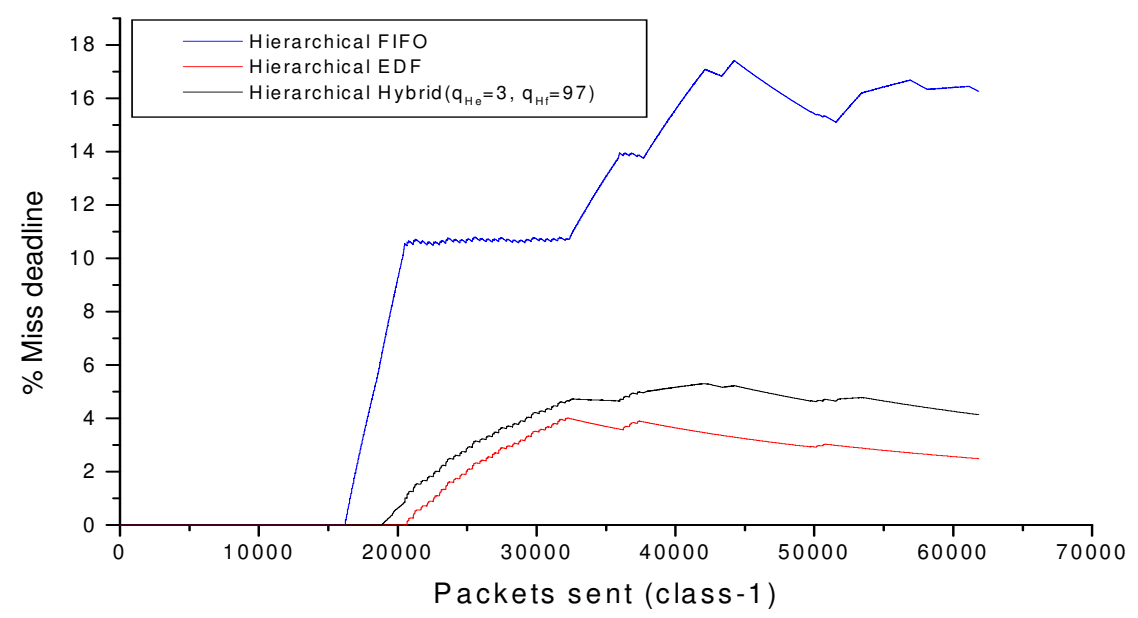

Figure 3. Comparison Hierarchical FIFO, Hierarchical EDF and Hierarchical Hybrid EDF/FIFO with $\mathrm{q}_{\mathrm{He}}=3$ and $\mathrm{q}_{\mathrm{Hf}}=97$ 


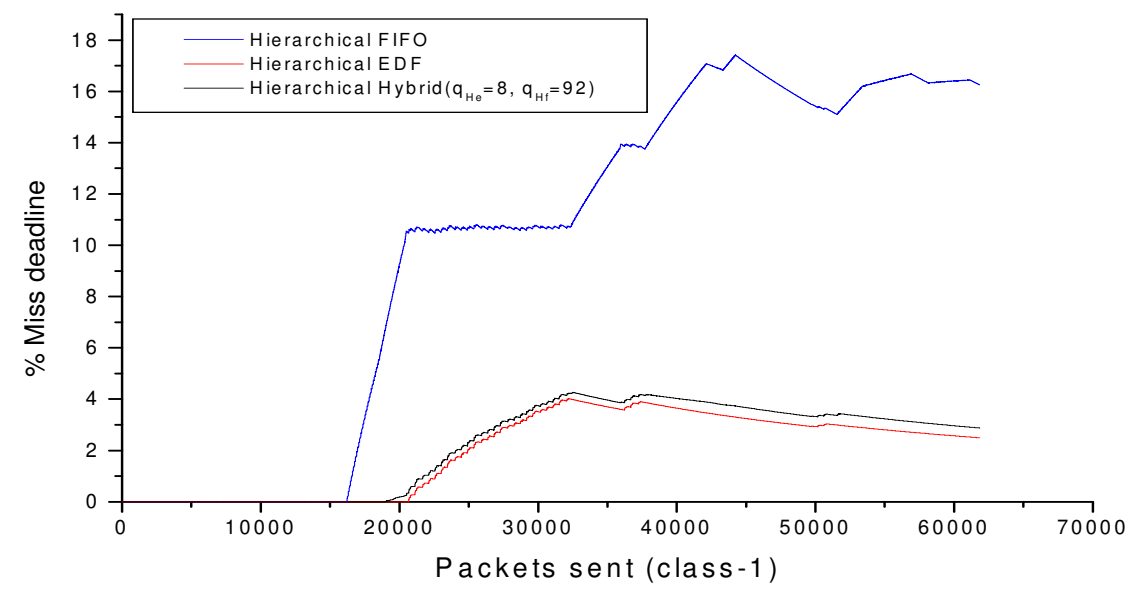

Figure 4. Comparison Hierarchical FIFO, Hierarchical EDF and Hierarchical Hybrid EDF/FIFO with $\mathrm{q}_{\mathrm{He}}=8$ and $\mathrm{q}_{\mathrm{Hf}}=92$

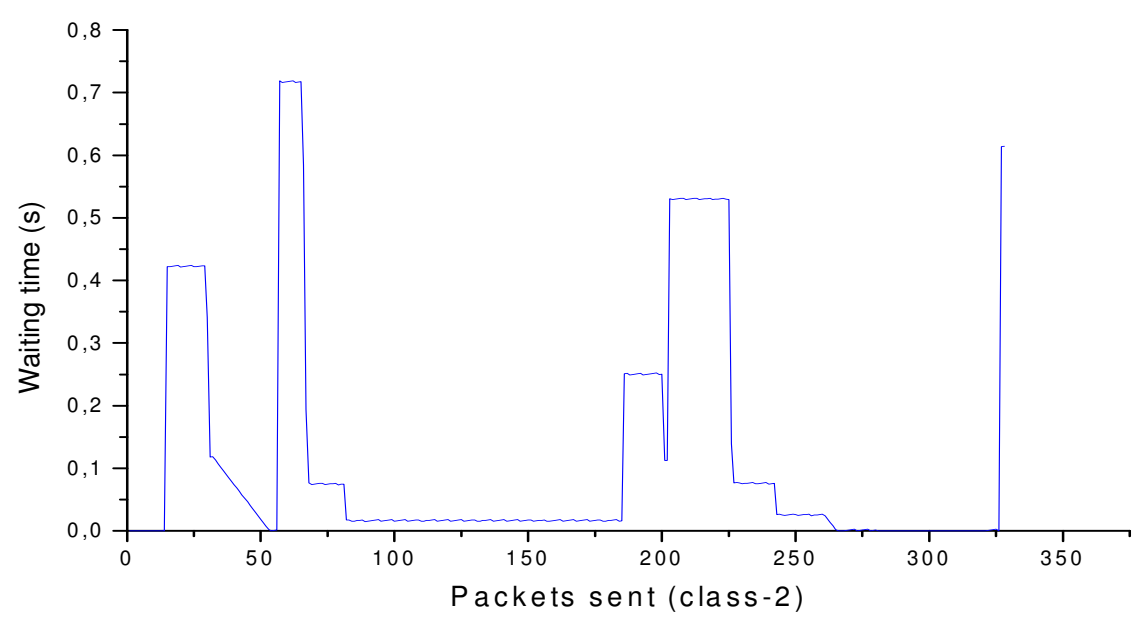

Figure 5. Waiting time of class-2 for the three scheduling algorithms

Figure 5 shows that the waiting time the packets of class- 2 is changed dramatically depending on the disponibility of packets the class-1

Finally, by analyzing the different figures above, we can conclude that the Hierarchical Hybrid EDF/FIFO scheduling shows a better performance of serving packet over heterogeneous network traffic with reduced complexity.

\section{Conclusions}

In this paper we presented a new scheduling approach for two flow classes. The class- 1 serves prioritized the real-time packets by hybrid policy EDF/FIFO, and the non real time class- 2 packets correspond to a lower priority and served by FIFO policy. The service discipline of the system is non-preemptive. The performance measures of class- 1 are the percentage of miss deadline of the packets, and for class-2 are the waiting time's packets. The main advantages of our hierarchical Hybrid EDF/FIFO queue resides in reducing the work complexity of the EDF 
algorithm, while maintaining to a great extent EDF's advantage in regarding packets' deadline respect while continuing to provide best effort service over heterogeneous network traffic environment. The simulation results showed that the Hierarchical Hybrid EDF/FIFO scheduler produces a better performance of packet serving over heterogeneous network traffic by achieving the minimum miss deadline's the packets.

The presented study will be continue to include extending the presented approach to multiserver systems and to compare the performance with other different scheduling such Round Robin RR, Weighted Fair Queuing (WFQ).

\section{REFERENCES}

[1] Andrew S. Tanenbaum, (2003) “Computer Networks”, Fourth Edition, Pearson Education.

[2] H. Zhang, (1995) "Service disciplines for guaranteed performance service in packet switching networks", proceedings of the IEEE, 83(10):1374-1399.

[3] P. Goyal, H. M. Vin, and H. Chen, (1996) "Start-time Fair Queuing: A scheduling algorithm for integrated services”, proceedings of the ACM-SIGCOMM '96, Palo Alto, CA, pp 157-168.

[4] R.W. Conway, W.L. Maxwell, and L.W. Miller, (1967) "Theory of Scheduling", AddisonWelsey.

[5] K. Jeffay, D. Stanat, and C. Martel, (1991) "On non-preemptive scheduling of periodic and sporadic tasks”, In IEEE Real-Time Systems Symposium, pages 129-139.

[6] J. Migge and A. Jean-Marie, (1998) "Timing analysis of real-time scheduling policies: A trajectoy based model”, In Tech. Report, INRIA, RR-3561.

[7] F. Chiussi, M. Gerla, and V. Sivaraman, (2000) "Traffic shaping for end-to-end delay guarantees with edf scheduling", In proceedings of International Workshop on Quality of Service (IWQoS).

[8] D. Ferrari and D. Verma, (1995) "A scheme for real-time channel establishment in wide-area networks”, IEEE Journal on Selected areas in communications, pages 368-379..

[9] L. George, P. Muhletahler, N. Rivierre, (1995) "Optimality and Non-preemptive Real-time Scheduling Revisited", Technical Report 2516, INRIA.

[10] GEORGIADIS.L, R.GUERIN, A.K.PAREKH, (1997) "Optimal Multiplexing on a Single Link : Delay and Buffer Requirements", Proceedings of the IEEE Transactions on Information Theory.

[11] V. Firoiu, J. Kurose, D. Towsley, (1997) "Efficient Admission Control for EDF Schedulers", Proceedings of IEEE INFOCOM, p. 310-317.

[12] R. Chipalkatti, J. Kurose, and D. Towsley, (1989) "Scheduling Policies for Real-Time and NonReal-Time Traffic in a Statistical Multiplexer”, In Proceedings of INFOCOM' 89, pages 774-783 vol.3.

[13] J. Peha and F. Tobagi, (1990) "Evaluation scheduling algorithms for traffic with heterogenous performance objectifs”, In Proceedings of GlobeCom'90, pages 21-27 vol.1, December 1990.

[14] V. Sivaraman, F. M. Chiussi, and M. Gerla, (2001) "End-to-end statistical delay service under GPS and EDF scheduling: A comparison study”, In Proceedings of IEEE INFOCOM'01, pages 1113-1122.

[15] A. Grilo, M. Macedo, and M. Nunes, (2003) “A scheduling algorithm for QoS support in IEEE802.11e networks”, In IEEE Wireless Communications, pages 36-43 vol.10.

[16] M. Andrews, (2000) "Probabilistic end-to-end delay bounds for earliest deadline first scheduling”, In Proceedings of IEEE INFOCOM'00, pages 603-612.

[17] M. D. Natale and A. Meschi, (2001) "Scheduling messages with earliest deadline techniques", In Journal Real-Time Syst., pages 255-285 vol.20. 
International Journal of Computer Science \& Information Technology (IJCSIT) Vol 3, No 4, August 2011

[18] F. Zhu, J.P. Lehoczky, J.P. Hansen, R. Rajkumar, (2005) "Diff-EDF: A Simple Mechanism for Differentiated EDF Services", Proceedings of the 11th IEEE Real-Time and Embedded Technology and Applications Symposium, pp. 268-277.

[19] O. C. Saadbouh, W. Fawaz and K. CHEN, (2006) "A Novel Approach for Real Time Flows Scheduling”, IEEE Globecom 2006, San Francisco, California, USA.

[20] P. Calyam and C.G. Lee, (2005) "Characterizing Voice and Video Traffic Behavior over the Internet". In International Symposium on Computer and Information Sciences (ISCIS), Istanbul, Turkey.

\section{Authors}

Rabie Barhoun is a researcher at the Department of Mathematics and Computer Science, Faculty of Science Ben M'sik, Hassan II Mohammedia University, Casablanca, Morocco. His current research interests are computer network, QoS and Protocol of communication.

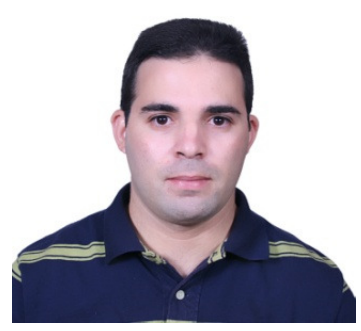

Namir Abdelwahed obtained the license in applied Mathematics in 1982; the PhD degree from the EMI School of Engineering, Rabat, Morocco in 1993. He is currently a full professor at the Faculty of Sciences of Ben M'sik, where he directs the Laboratory for Research in Laboratory of Information Technology and Modeling. His fields of interest are QoS, Telecommunication, and Protocol of communication. 